\title{
Boosting-based parse reranking with subtree features
}

\author{
Taku Kudo * Jun Suzuki Hideki Isozaki \\ NTT Communication Science Laboratories. \\ 2-4 Hikaridai, Seika-cho, Soraku, Kyoto, Japan \\ \{taku, jun, isozaki\}@cslab.kecl.ntt.co.jp
}

\begin{abstract}
This paper introduces a new application of boosting for parse reranking. Several parsers have been proposed that utilize the all-subtrees representation (e.g., tree kernel and data oriented parsing). This paper argues that such an all-subtrees representation is extremely redundant and a comparable accuracy can be achieved using just a small set of subtrees. We show how the boosting algorithm can be applied to the all-subtrees representation and how it selects a small and relevant feature set efficiently. Two experiments on parse reranking show that our method achieves comparable or even better performance than kernel methods and also improves the testing efficiency.
\end{abstract}

\section{Introduction}

Recent work on statistical natural language parsing and tagging has explored discriminative techniques. One of the novel discriminative approaches is reranking, where discriminative machine learning algorithms are used to rerank the $n$-best outputs of generative or conditional parsers. The discriminative reranking methods allow us to incorporate various kinds of features to distinguish the correct parse tree from all other candidates.

With such feature design flexibility, it is nontrivial to employ an appropriate feature set that has a good discriminative ability for parse reranking. In early studies, feature sets were given heuristically by simply preparing task-dependent feature templates (Collins, 2000; Collins, 2002). These ad-hoc solutions might provide us with reasonable levels of per-

\footnotetext{
${ }^{*}$ Currently, Google Japan Inc., taku@ google.com
}

formance. However, they are highly task dependent and require careful design to create the optimal feature set for each task. Kernel methods offer an elegant solution to these problems. They can work on a potentially huge or even infinite number of features without a loss of generalization. The best known kernel for modeling a tree is the tree kernel (Collins and Duffy, 2002), which argues that a feature vector is implicitly composed of the counts of subtrees. Although kernel methods are general and can cover almost all useful features, the set of subtrees that is used is extremely redundant. The main question addressed in this paper concerns whether it is possible to achieve a comparable or even better accuracy using just a small and non-redundant set of subtrees.

In this paper, we present a new application of boosting for parse reranking. While tree kernel implicitly uses the all-subtrees representation, our boosting algorithm uses it explicitly. Although this set-up makes the feature space large, the $l_{1}$-norm regularization achived by boosting automatically selects a small and relevant feature set. Such a small feature set is useful in practice, as it is interpretable and makes the parsing (reranking) time faster. We also incorporate a variant of the branch-and-bound technique to achieve efficient feature selection in each boosting iteration.

\section{General setting of parse reranking}

We describe the general setting of parse reranking.

- Training data $T$ is a set of input/output pairs, e.g., $T=\left\{\left\langle\mathbf{x}_{1}, \mathbf{y}_{1}\right\rangle, \ldots,\left\langle\mathbf{x}_{L}, \mathbf{y}_{L}\right\rangle\right\}$, where $\mathbf{x}_{i}$ is an input sentence, and $\mathbf{y}_{i}$ is a correct parse associated with the sentence $\mathbf{x}_{i}$.

- Let $\mathcal{Y}(\mathbf{x})$ be a function that returns a set of candi- 
date parse trees for a particular sentence $\mathbf{x}$.

- We assume that $\mathcal{Y}\left(\mathbf{x}_{i}\right)$ contains the correct parse tree $\mathbf{y}_{i}$, i.e., $\mathbf{y}_{i} \in \mathcal{Y}\left(\mathbf{x}_{i}\right)^{*}$

- Let $\Phi(\mathbf{y}) \in \mathbb{R}^{d}$ be a feature function that maps the given parse tree $\mathbf{y}$ into $\mathbb{R}^{d}$ space. $\mathbf{w} \in \mathbb{R}^{d}$ is a parameter vector of the model. The output parse $\hat{\mathbf{y}}$ of this model on input sentence $\mathbf{x}$ is given as: $\hat{\mathbf{y}}=\operatorname{argmax}_{\mathbf{y} \in \mathcal{Y}(\mathbf{x})} \mathbf{w} \cdot \Phi(\mathbf{y})$.

There are two questions as regards this formulation. One is how to set the parameters $\mathbf{w}$, and the other is how to design the feature function $\Phi(\mathbf{y})$. We briefly describe the well-known solutions to these two problems in the next subsections.

\subsection{Parameter estimation}

We usually adopt a general loss function $\operatorname{Loss}(\mathbf{w})$, and set the parameters $\mathbf{w}$ that minimize the loss, i.e., $\hat{\mathbf{w}}=\operatorname{argmin}_{\mathbf{w} \in \mathbb{R}^{d}} \operatorname{Loss}(\mathbf{w})$. Generally, the loss function has the following form:

$$
\operatorname{Loss}(\mathbf{w})=\sum_{i=1}^{L} L\left(\mathbf{w}, \Phi\left(\mathbf{y}_{i}\right), \mathbf{x}_{i}\right),
$$

where $L\left(\mathbf{w}, \Phi\left(\mathbf{y}_{i}\right), \mathbf{x}_{i}\right)$ is an arbitrary loss function. We can design a variety of parameter estimation methods by changing the loss function. The following three loss functions, LogLoss, HingeLoss, and BoostLoss, have been widely used in parse reranking tasks.

$$
\begin{aligned}
\text { LogLoss } & =-\log \sum_{\mathbf{y} \in \mathcal{Y}\left(\mathbf{x}_{i}\right)} \exp \mathbf{w} \cdot\left[\Phi\left(\mathbf{y}_{i}\right)-\Phi(\mathbf{y})\right] \\
\text { HingeLoss } & =\sum_{\mathbf{y} \in \mathcal{Y}\left(\mathbf{x}_{i}\right)} \max \left(0,1-\mathbf{w} \cdot\left[\Phi\left(\mathbf{y}_{i}\right)-\Phi(\mathbf{y})\right]\right) \\
\text { BoostLos } & =\sum_{\mathbf{y} \in \mathcal{Y}\left(\mathbf{x}_{i}\right)} \exp -\mathbf{w} \cdot\left[\Phi\left(\mathbf{y}_{i}\right)-\Phi(\mathbf{y})\right]
\end{aligned}
$$

LogLoss is based on the standard maximum likelihood optimization, and is used with maximum entropy models. HingeLoss captures the errors only when $\left.\mathbf{w} \cdot\left[\Phi\left(\mathbf{y}_{i}\right)-\Phi(\mathbf{y})\right]\right)<1$. This loss is closely related to the maximum margin strategy in SVMs (Vapnik, 1998). BoostLoss is analogous to the boosting algorithm and is used in (Collins, 2000; Collins, 2002).

\footnotetext{
${ }^{*}$ In the real setting, we cannot assume this condition. In this case, we select the parse tree $\hat{\mathbf{y}}$ that is the most similar to $\mathbf{y}_{i}$ and take $\hat{\mathbf{y}}$ as the correct parse tree $\mathbf{y}_{i}$.
}

\subsection{Definition of feature function}

It is non-trivial to define an appropriate feature function $\Phi(\mathbf{y})$ that has a good ability to distinguish the correct parse $\mathbf{y}_{i}$ from all other candidates

In early studies, the feature functions were given heuristically by simply preparing feature templates (Collins, 2000; Collins, 2002). However, such heuristic selections are task dependent and would not cover all useful features that contribute to overall accuracy.

When we select the special family of loss functions, the problem can be reduced to a dual form that depends only on the inner products of two instances $\Phi\left(\mathbf{y}_{1}\right) \cdot \Phi\left(\mathbf{y}_{2}\right)$. This property is important as we can use a kernel trick and we do not need to provide an explicit feature function. For example, tree kernel (Collins and Duffy, 2002), one of the convolution kernels, implicitly maps the instance represented in a tree into all-subtrees space. Even though the feature space is large, inner products under this feature space can be calculated efficiently using dynamic programming. Tree kernel is more general than feature templates since it can use the all-subtrees representation without loss of efficiency.

\section{RankBoost with subtree features}

A simple question related to kernel-based parse reranking asks whether all subtrees are really needed to construct the final parameters w. Suppose we have two large trees $t$ and $t^{\prime}$, where $t^{\prime}$ is simply generated by attaching a single node to $t$. In most cases, these two trees yield an almost equivalent discriminative ability, since they are very similar and highly correlated with each other. Even when we exploit all subtrees, most of them are extremely redundant.

The motivation of this paper is based on the above observation. We think that only a small set of subtrees is needed to express the final parameters. A compact, non-redundant, and highly relevant feature set is useful in practice, as it is interpretable and increases the parsing (reranking) speed.

To realize this goal, we propose a new boostingbased reranking algorithm based on the all-subtrees representation. First, we describe the architecture of our reranking method. Second, we show a connection between boosting and SVMs, and describe how the algorithm realizes the sparse feature representa- 


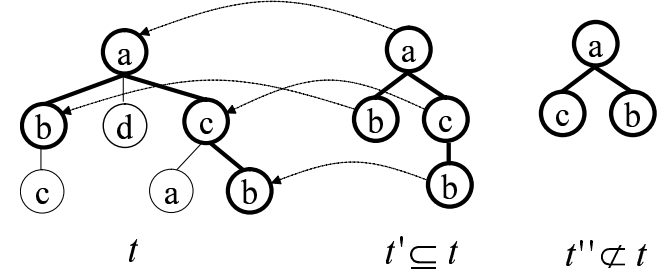

Figure 1: Labeled ordered tree and subtree relation tion described above.

\subsection{Preliminaries}

Let us introduce a labeled ordered tree (or simply 'tree'), its definition and notations, first.

Definition 1 Labeled ordered tree (Tree)

A labeled ordered tree is a tree where each node is associated with a label and is ordered among its siblings, that is, there is a first child, second child, third child, etc.

\section{Definition 2 Subtree}

Let $t$ and $u$ be labeled ordered trees. We say that $t$ matches $u$, or $t$ is a subtree of $u(t \subseteq u)$, if there is a one-to-one function $\psi$ from nodes in to to $u$, satisfying the conditions: (1) $\psi$ preserves the parent-daughter relation, (2) $\psi$ preserves the sibling relation, (3) $\psi$ preserves the labels.

We denote the number of nodes in $t$ as $|t|$. Figure 1 shows an example of a labeled ordered tree and its subtree and non-subtree.

\subsection{Feature space given by subtrees}

We first assume that a parse tree $\mathbf{y}$ is represented in a labeled ordered tree. Note that the outputs of partof-speech tagging, shallow parsing, and dependency analysis can be modeled as labeled ordered trees.

The feature set $\mathcal{F}$ consists of all subtrees seen in the training data, i.e.,

$$
\mathcal{F}=\cup_{i, \mathbf{y} \in \mathcal{Y}\left(\mathbf{x}_{i}\right)}\{t \mid t \subseteq \mathbf{y}\} .
$$

The feature mapping $\Phi(\mathbf{y})$ is then given by letting the existence of a tree $t$ be a single dimension, i.e.,

$$
\Phi(\mathbf{y})=\left\{I\left(t_{1} \subseteq \mathbf{y}\right), \ldots, I\left(t_{m} \subseteq \mathbf{y}\right)\right\} \in\{0,1\}^{m},
$$

where $I(\cdot)$ is the indicator function, $m=|\mathcal{F}|$, and $\left\{t_{1}, \ldots, t_{m}\right\} \in \mathcal{F}$. The feature space is essentially the same as that of tree kernel ${ }^{\dagger}$

\footnotetext{
${ }^{\dagger}$ Strictly speaking, tree kernel uses the cardinality of each subtree
}

\subsection{RankBoost algorithm}

The parameter estimation method we adopt is a variant of the RankBoost algorithm introduced in (Freund et al., 2003). Collins et al. used RankBoost to parse reranking tasks (Collins, 2000; Collins, 2002). The algorithm proceeds for $K$ iterations and tries to minimize the BoostLoss for given training data ${ }^{\ddagger}$. At each iteration, a single feature (hypothesis) is chosen, and its weight is updated.

Suppose we have current parameters:

$$
\mathbf{w}=\left\{w_{1}, w_{2}, \ldots, w_{m}\right\} \in \mathbb{R}^{m} .
$$

New parameters $\mathbf{w}^{*}\langle k, \delta\rangle \in \mathbb{R}^{m}$ are then given by selecting a single feature $k$ and updating the weight through an increment $\delta$ :

$$
\mathbf{w}^{*}{ }_{\langle k, \delta\rangle}=\left\{w_{1}, w_{2}, \ldots, w_{k}+\delta, \ldots, w_{m}\right\} .
$$

After the update, the new loss is given:

$$
\operatorname{Loss}\left(\mathbf{w}^{*}\langle k, \delta\rangle\right)=\sum_{i, \mathbf{y} \in \mathcal{Y}\left(\mathbf{x}_{i}\right)} \exp -\mathbf{w}_{\langle k, \delta\rangle}^{*} \cdot\left[\Phi\left(\mathbf{y}_{i}\right)-\Phi(\mathbf{y})\right] .
$$

The RankBoost algorithm iteratively selects the optimal pair $\langle\hat{k}, \hat{\delta}\rangle$ that minimizes the loss, i.e.,

$$
\langle\hat{k}, \hat{\delta}\rangle=\underset{\langle k, \delta\rangle}{\operatorname{argmin}} \operatorname{Loss}\left(\mathbf{w}^{*}{ }_{\langle k, \delta\rangle}\right) .
$$

By setting the differential of (1) at 0 , the following optimal solutions are obtained:

$$
\hat{k}=\underset{k=1, \ldots, m}{\operatorname{argmax}} \sqrt{W_{k}^{+}}-\sqrt{W_{k}^{-}}, \text {and } \delta=\frac{1}{2} \log \frac{W_{\hat{k}}^{+}}{W_{\hat{k}}^{-}},
$$

where $W_{k}^{b}=\sum_{i, \mathbf{y} \in \mathcal{Y}\left(\mathbf{x}_{i}\right)} D\left(\mathbf{y}_{i}, \mathbf{y}\right) \cdot I\left[I\left(t_{k} \subseteq \mathbf{y}_{i}\right)-\right.$ $\left.I\left(t_{k} \subseteq \mathbf{y}\right)=b\right], b \in\{+1,-1\}$, and $D\left(\mathbf{y}_{i}, \mathbf{y}\right)=$ $\exp \left(-\mathbf{w} \cdot\left[\Phi\left(\mathbf{y}_{i}\right)-\Phi(\mathbf{y})\right]\right)$.

Following (Freund et al., 2003; Collins, 2000), we introduce smoothing to prevent the case when either $W_{k}^{+}$or $W_{k}^{-}$is $0 \AA$ :

$\delta=\frac{1}{2} \log \frac{W_{\hat{k}}^{+}+\epsilon Z}{W_{\hat{k}}^{-}+\epsilon Z}$, where $Z=\sum_{i, \mathbf{y} \in \mathcal{Y}\left(\mathbf{x}_{i}\right)} D\left(\mathbf{y}_{i}, \mathbf{y}\right)$ and $\epsilon \in \mathbb{R}^{+}$.

The function $\mathcal{Y}(\mathbf{x})$ is usually performed by a probabilistic history-based parser, which can output not only a parse tree but the log probability of the

\footnotetext{
${ }^{\ddagger}$ In our experiments, optimal settings for $K$ were selected by using development data.

${ }^{\S}$ For simplicity, we fix $\epsilon$ at 0.001 in all our experiments.
} 
tree. We incorporate the log probability into the reranking by using it as a feature:

$$
\begin{aligned}
\Phi(\mathbf{y}) & =\left\{L(\mathbf{y}), I\left(t_{1} \subseteq \mathbf{y}\right), \ldots, I\left(t_{m} \subseteq \mathbf{y}\right)\right\}, \text { and } \\
\mathbf{w} & =\left\{w_{0}, w_{1}, w_{2}, \ldots, w_{m}\right\}
\end{aligned}
$$

where $L(\mathbf{y})$ is the log probability of a tree $\mathbf{y}$ under the base parser and $w_{0}$ is the parameter of $L(\mathbf{y})$. Note that the update algorithm (2) does not allow us to calculate the parameter $w_{0}$, since (2) is restricted to binary features. To prevent this problem, we use the approximation technique introduced in (Freund et al., 2003).

\subsection{Sparse feature representation}

Recent studies (Schapire et al., 1997; Rätsch, 2001) have shown that both boosting and SVMs (Vapnik, 1998) work according to similar strategies: constructing optimal parameters $\mathbf{w}$ that maximize the smallest margin between positive and negative examples. The critical difference is the definition of margin or the way they regularize the vector $\mathbf{w}$. (Rätsch, 2001) shows that the iterative feature selection performed in boosting asymptotically realizes an $l_{1}$-norm $\|\mathbf{w}\|_{1}$ regularization. In contrast, it is well known that SVMs are reformulated as an $l_{2}$ norm $\|\mathbf{w}\|_{2}$ regularized algorithm.

The relationship between two regularizations has been studied in the machine learning community. (Perkins et al., 2003) reported that $l_{1}$-norm should be chosen for a problem where most given features are irrelevant. On the other hand, $l_{2}$-norm should be chosen when most given features are relevant. An advantage of the $l_{1}$-norm regularizer is that it often leads to sparse solutions where most $w_{k}$ are exactly 0 . The features assigned zero weight are thought to be irrelevant features as regards classifications.

The $l_{1}$-norm regularization is useful for our setting, since most features (subtrees) are redundant and irrelevant, and these redundant features are automatically eliminated.

\section{Efficient Computation}

In each boosting iteration, we have to solve the following optimization problem:

$$
\begin{aligned}
& \hat{k}=\underset{k=1, \ldots, m}{\operatorname{argmax}} \operatorname{gain}\left(t_{k}\right), \\
& \text { where } \operatorname{gain}\left(t_{k}\right)=\left|\sqrt{W_{k}^{+}}-\sqrt{W_{k}^{-}}\right| .
\end{aligned}
$$

It is non-trivial to find the optimal tree $t_{\hat{k}}$ that maximizes gain $\left(t_{k}\right)$, since the number of subtrees is exponential to its size. In fact, the problem is known to be NP-hard (Yang, 2004). However, in real applications, the problem is manageable, since the maximum number of subtrees is usually bounded by a constant. To solve the problem efficiently, we now adopt a variant of the branch-and-bound algorithm, similar to that described in (Kudo and Matsumoto, 2004)

\subsection{Efficient Enumeration of Trees}

Abe and Zaki independently proposed an efficient method, rightmost-extension, for enumerating all subtrees from a given tree (Abe et al., 2002; Zaki, 2002). First, the algorithm starts with a set of trees consisting of single nodes, and then expands a given tree of size $(n-1)$ by attaching a new node to it to obtain trees of size $n$. However, it would be inefficient to expand nodes at arbitrary positions of the tree, as duplicated enumeration is inevitable. The algorithm, rightmost extension, avoids such duplicated enumerations by restricting the position of attachment. Here we give the definition of rightmost extension to describe this restriction in detail.

Definition 3 Rightmost Extension (Abe et al., 2002; Zaki, 2002)

Let $t$ and $t^{\prime}$ be labeled ordered trees. We say $t^{\prime}$ is a rightmost extension of $t$, if and only if $t$ and $t^{\prime}$ satisfy the following three conditions:

(1) $t^{\prime}$ is created by adding a single node to $t$, (i.e., $t \subset t^{\prime}$ and $\left.|t|+1=\left|t^{\prime}\right|\right)$.

(2) A node is added to a node existing on the unique path from the root to the rightmost leaf (rightmostpath) in $t$.

(3) A node is added as the rightmost sibling.

Consider Figure 2, which illustrates example tree $t$ with labels drawn from the set $\mathcal{L}=\{a, b, c\}$. For the sake of convenience, each node in this figure has its original number (depth-first enumeration). The rightmost-path of the tree $t$ is $(a(c(b)))$, and it occurs at positions 1,4 and 6 respectively. The set of rightmost extended trees is then enumerated by simply adding a single node to a node on the rightmost path. Since there are three nodes on the rightmost path and the size of the label set is $3(=|\mathcal{L}|)$, a to- 


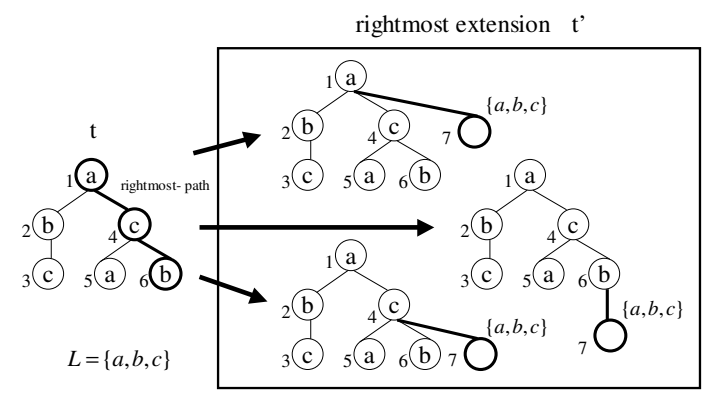

Figure 2: Rightmost extension

tal of 9 trees are enumerated from the original tree $t$. By repeating the rightmost-extension process recursively, we can create a search space in which all trees drawn from the set $\mathcal{L}$ are enumerated.

\subsection{Pruning}

Rightmost extension defines a canonical search space in which we can enumerate all subtrees from a given set of trees. Here we consider an upper bound of the gain that allows subspace pruning in this canonical search space. The following observation provides a convenient way of computing an upper bound of the $\operatorname{gain}\left(t_{k}\right)$ for any super-tree $t_{k^{\prime}}$ of $t_{k}$.

Observation 1 Upper bound of the gain $\left(t_{k}\right)$ For any $t_{k^{\prime}} \supseteq t_{k}$, the gain of $t_{k^{\prime}}$ is bounded by $\mu\left(t_{k}\right)$ :

$$
\begin{aligned}
\operatorname{gain}\left(t_{k^{\prime}}\right) & =\sqrt{W_{k^{\prime}}^{+}}-\sqrt{W_{k^{\prime}}^{-}} \\
& \leq \max \left(\sqrt{W_{k^{\prime}}^{+}}, \sqrt{W_{k^{\prime}}^{-}}\right) \\
& \leq \max \left(\sqrt{W_{k}^{+}}, \sqrt{W_{k}^{-}}\right)=\mu\left(t_{k}\right), \\
\text { since } & t_{k^{\prime}} \supseteq t_{k} \Rightarrow W_{k^{\prime}}^{b} \leq W_{k}^{b}, b \in\{+1,-1\} .
\end{aligned}
$$

We can efficiently prune the search space spanned by the rightmost extension using the upper bound of gain $\mu(t)$. During the traverse of the subtree lattice built by the recursive process of rightmost extension, we always maintain the temporally suboptimal gain $\tau$ of all the previously calculated gains. If $\mu(t)<\tau$, the gain of any super-tree $t^{\prime} \supseteq t$ is no greater than $\tau$, and therefore we can safely prune the search space spanned from the subtree $t$. In contrast, if $\mu(t) \geq \tau$, we cannot prune this space, since there might be a super-tree $t^{\prime} \supseteq t$ such that gain $\left(t^{\prime}\right) \geq \tau$.

\subsection{Ad-hoc techniques}

In real applications, we also employ the following practical methods to reduce the training costs.
- Size constraint

Larger trees are usually less effective to discrimination. Thus, we give a size threshold $s$, and use subtrees whose size is no greater than $s$. This constraint is easily realized by controlling the rightmost extension according to the size of the trees.

- Frequency constraint

The frequency-based cut-off has been widely used in feature selections. We employ a frequency threshold $f$, and use subtrees seen on at least one parse for at least $f$ different sentences. Note that a similar branch-and-bound technique can also be applied to the cut-off. When we find that the frequency of a tree $t$ is no greater than $f$, we can safely prune the space spanned from $t$ as the frequencies of any super-trees $t^{\prime} \supseteq t$ are also no greater than $f$.

- Pseudo iterations

After several 5- or 10-iterations of boosting, we alternately perform 100- or 300 pseudo iterations, in which the optimal feature (subtree) is selected from the cache that maintains the features explored in the previous iterations. The idea is based on our observation that a feature in the cache tends to be reused as the number of boosting iterations increases. Pseudo iterations converge very fast, and help the branch-and-bound algorithm find new features that are not in the cache.

\section{Experiments}

\subsection{Parsing Wall Street Journal Text}

In our experiments, we used the same data set that used in (Collins, 2000). Sections 2-21 of the Penn Treebank were used as training data, and section 23 was used as test data. The training data contains about 40,000 sentences, each of which has an average of 27 distinct parses. Of the 40,000 training sentences, the first 36,000 sentences were used to perform the RankBoost algorithm. The remaining 4,000 sentences were used as development data. Model2 of (Collins, 1999) was used to parse both the training and test data.

To capture the lexical information of the parse trees, we did not use a standard CFG tree but a lexicalized-CFG tree where each non-terminal node has an extra lexical node labeled with the head word of the constituent. Figure 3 shows an example of the lexicalized-CFG tree used in our experiments. The 


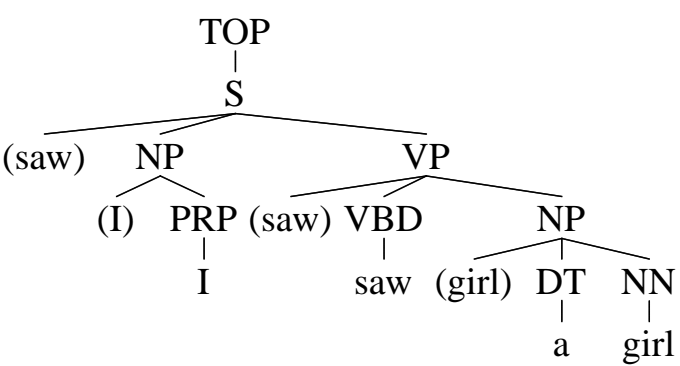

Figure 3: Lexicalized CFG tree for WSJ parsing head word, e.g., (saw), is put as a leftmost constituent

size parameter $s$ and frequency parameter $f$ were experimentally set at 6 and 10, respectively. As the data set is very large, it is difficult to employ the experiments with more unrestricted parameters.

Table 1 lists results on test data for the Model 2 of (Collins, 1999), for several previous studies, and for our best model. We achieve recall and precision of $89.3 / \% 89.6 \%$ and $89.9 \% / 90.1 \%$ for sentences with $\leq 100$ words and $\leq 40$ words, respectively. The method shows a $1.2 \%$ absolute improvement in average precision and recall (from $88.2 \%$ to $89.4 \%$ for sentences $\leq 100$ words), a $10.1 \%$ relative reduction in error. (Collins, 2000) achieved 89.6\%/89.9\% recall and precision for the same datasets (sentences $\leq 100$ words) using boosting and manually constructed features. (Charniak, 2000) extends PCFG and achieves similar performance to (Collins, 2000). The tree kernel method of (Collins and Duffy, 2002) uses the all-subtrees representation and achieves $88.6 \% / 88.9 \%$ recall and precision, which are slightly worse than the results obtained with our model. (Bod, 2001) also uses the all-subtrees representation with a very different parameter estimation method, and realizes $90.06 \% / 90.08 \%$ recall and precision for sentences of $\leq 40$ words.

\subsection{Shallow Parsing}

We used the same data set as the CoNLL 2000 shared task (Tjong Kim Sang and Buchholz, 2000). Sections 15-18 of the Penn Treebank were used as training data, and section 20 was used as test data.

As a baseline model, we used a shallow parser based on Conditional Random Fields (CRFs), very similar to that described in (Sha and Pereira, 2003). CRFs have shown remarkable results in a number of tagging and chunking tasks in NLP. $n$-best outputs were obtained by a combination of forward

\begin{tabular}{|c|c|c|c|c|c|}
\hline \multirow{2}{*}{ MODEL } & \multicolumn{5}{|c|}{$\leq 40$ Words (2245 sentences) } \\
\cline { 2 - 6 } & LR & LP & CBs & 0 CBs & 2 CBs \\
\hline CO99 & $88.5 \%$ & $88.7 \%$ & 0.92 & $66.7 \%$ & $87.1 \%$ \\
CH00 & $90.1 \%$ & $90.1 \%$ & 0.74 & $70.1 \%$ & $89.6 \%$ \\
CO00 & $90.1 \%$ & $90.4 \%$ & 0.74 & $70.3 \%$ & $89.6 \%$ \\
CO02 & $89.1 \%$ & $89.4 \%$ & 0.85 & $69.3 \%$ & $88.2 \%$ \\
Boosting & $89.9 \%$ & $90.1 \%$ & 0.77 & $70.5 \%$ & $89.4 \%$ \\
\hline \hline MODEL & $\leq 100$ Words (2416 sentences) \\
\cline { 2 - 6 } & LR & LP & CBs & 0 CBs & 2 CBs \\
\hline CO99 & $88.1 \%$ & $88.3 \%$ & 1.06 & $64.0 \%$ & $85.1 \%$ \\
CH00 & $89.6 \%$ & $89.5 \%$ & 0.88 & $67.6 \%$ & $87.7 \%$ \\
CO00 & $89.6 \%$ & $89.9 \%$ & 0.87 & $68.3 \%$ & $87.7 \%$ \\
CO02 & $88.6 \%$ & $88.9 \%$ & 0.99 & $66.5 \%$ & $86.3 \%$ \\
Boosting & $89.3 \%$ & $89.6 \%$ & 0.90 & $67.9 \%$ & $87.5 \%$ \\
\hline
\end{tabular}

Table 1: Results for section 23 of the WSJ Treebank $\mathrm{LR} / \mathrm{LP}=$ labeled recall/precision. $\mathrm{CBs}$ is the average number of cross brackets per sentence. $0 \mathrm{CBs}$, and $2 \mathrm{CBs}$ are the percentage of sentences with 0 or $\leq 2$ crossing brackets, respectively. COL99 $=$ Model 2 of (Collins, 1999). CH00 $=($ Charniak, 2000), CO00 $=($ Collins, 2000). CO02 $=($ Collins and Duffy, 2002).

Viterbi search and backward $A^{*}$ search. Note that this search algorithm yields optimal $n$-best results in terms of the CRFs score. Each sentence has at most 20 distinct parses. The log probability from the CRFs shallow parser was incorporated into the reranking. Following (Collins, 2000), the training set was split into 5 portions, and the CRFs shallow parser was trained on $4 / 5$ of the data, then used to decode the remaining $1 / 5$. The outputs of the base parser, which consist of base phrases, were converted into right-branching trees by assuming that two adjacent base phrases are in a parent-child relationship. Figure 4 shows an example of the tree for shallow parsing task. We also put two virtual nodes, left/right boundaries, to capture local transitions. The size parameter $s$ and frequency parameter $f$ were experimentally set at 6 and 5 , respectively.

Table 2 lists results on test data for the baseline CRFs parser, for several previous studies, and for our best model. Our model achieves a 94.12 Fmeasure, and outperforms the baseline CRFs parser and the SVMs parser (Kudo and Matsumoto, 2001). (Zhang et al., 2002) reported a higher F-measure with a generalized winnow using additional linguistic features. The accuracy of our model is very similar to that of (Zhang et al., 2002) without using such additional features. Table 3 shows the results for our best model per chunk type. 


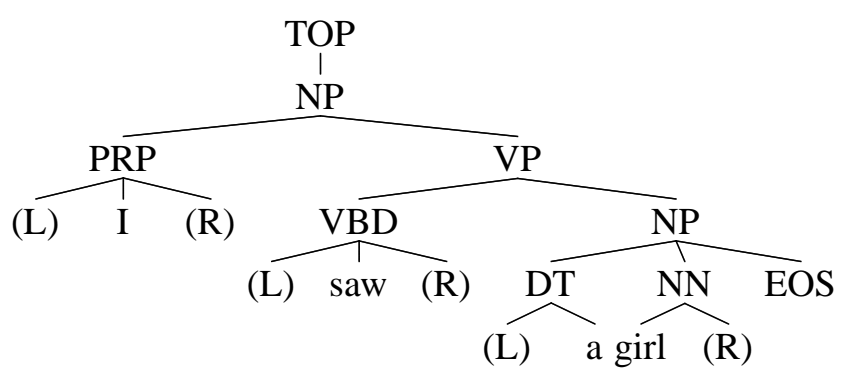

Figure 4: Tree representation for shallow parsing Represented in a right-branching tree with two virtual nodes

\begin{tabular}{|l|r|}
\hline MODEL & $F_{\beta=1}$ \\
\hline CRFs (baseline) & 93.76 \\
8 SVMs-voting (Kudo and Matsumoto, 2001) & 93.91 \\
RW + linguistic features (Zhang et al., 2002) & 94.17 \\
Boosting (our model) & 94.12 \\
\hline
\end{tabular}

Table 2: Results of shallow parsing

$F_{\beta=1}$ is the harmonic mean of precision and recall.

\section{Discussion}

\subsection{Interpretablity and Efficiency}

The numbers of active (non-zero) features selected by boosting are around 8,000 and 3,000 in the WSJ parsing and shallow parsing, respectively. Although almost all the subtrees are used as feature candidates, boosting selects a small and highly relevant subset of features. When we explicitly enumerate the subtrees used in tree kernel, the number of active features might amount to millions or more. Note that the accuracies under such sparse feature spaces are still comparable to those obtained with tree kernel. This result supports our first intuition that we do not always need all the subtrees to construct the parameters.

The sparse feature representations are useful in practice as they allow us to analyze what kinds of features are relevant. Table 4 shows examples of active features along with their weights $w_{k}$. In the shallow parsing tasks, subordinate phrases (SBAR) are difficult to analyze without seeing long dependencies. Subordinate phrases usually precede a sentence (NP and VP). However, Markov-based shallow parsers, such as MEMM or CRFs, cannot capture such a long dependency. Our model automatically selects useful subtrees to obtain an improvement on subordinate phrases. It is interesting that the

\begin{tabular}{|l|c|c|c|}
\hline & Precision & Recall & $F_{\beta=1}$ \\
\hline ADJP & $80.35 \%$ & $73.41 \%$ & 76.72 \\
ADVP & $83.88 \%$ & $82.33 \%$ & 83.10 \\
CONJP & $42.86 \%$ & $66.67 \%$ & 52.17 \\
INTJ & $50.00 \%$ & $50.00 \%$ & 50.00 \\
LST & $0.00 \%$ & $0.00 \%$ & 0.00 \\
NP & $94.45 \%$ & $94.36 \%$ & 94.41 \\
PP & $97.24 \%$ & $98.07 \%$ & 97.65 \\
PRT & $76.92 \%$ & $75.47 \%$ & 76.19 \\
SBAR & $90.70 \%$ & $89.35 \%$ & 90.02 \\
VP & $93.95 \%$ & $94.72 \%$ & 94.33 \\
\hline Overall & $94.11 \%$ & $94.13 \%$ & 94.12 \\
\hline
\end{tabular}

Table 3: Results of shallow parsing per chunk type tree (SBAR(IN(for))(NP(VP(TO)))) has a large positive weight, while the tree $(\operatorname{SBAR}((\operatorname{IN}($ for $))(\mathrm{NP}(\mathrm{O}))))$ has a negative weight. The improvement on subordinate phrases is considerable. We achieve $19 \%$ of the relative error reduction for subordinate phrase (from 87.68 to 90.02 in F-measure)

The testing speed of our model is much higher than that of other models. The speeds of reranking for WSJ parsing and shallow parsing are 0.055 sec./sent. and $0.042 \mathrm{sec} . /$ sent. respectively, which are fast enough for real applications $₫$.

\subsection{Relationship to previous work}

Tree kernel uses the all-subtrees representation not explicitly but implicitly by reducing the problem to the calculation of the inner-products of two trees. The implicit calculation yields a practical computation in training. However, in testing, kernel methods require a number of kernel evaluations, which are too heavy to allow us to realize real applications. Moreover, tree kernel needs to incorporate a decay factor to downweight the contribution of larger subtrees. It is non-trivial to set the optimal decay factor as the accuracies are sensitive to its selection.

Similar to our model, data oriented parsing (DOP) methods (Bod, 1998) deal with the all-subtrees representation explicitly. Since the exact computation of scores for DOP is NP-complete, several approximations are employed to perform an efficient parsing. The critical difference between our model and DOP is that our model leads to an extremely sparse solution and automatically eliminates redundant subtrees. With the DOP methods, (Bod, 2001) also employs constraints (e.g., depth of subtrees) to

\footnotetext{
${ }^{9}$ We ran these tests on a Linux PC with Pentium 4 3.2 Ghz.
} 


\begin{tabular}{|c|c|}
\hline \multicolumn{2}{|r|}{ WSJ parsing } \\
\hline$w$ & active trees that contain the word "in" \\
\hline 0.3864 & $(\mathrm{VP}(\mathrm{NP}(\mathrm{NNS}$ (plants)))(PP(in))) \\
\hline 0.3326 & $(\mathrm{VP}(\mathrm{VP}(\mathrm{PP})(\mathrm{PP}(\mathrm{in})))(\mathrm{VP}))$ \\
\hline 0.2196 & $(\mathrm{NP}(\mathrm{VP}(\mathrm{VP}(\mathrm{PP})(\mathrm{PP}(\mathrm{in})))))$ \\
\hline 0.1748 & $(\mathrm{~S}(\mathrm{NP}(\mathrm{NNP}))(\mathrm{PP}(\mathrm{in})(\mathrm{NP})))$ \\
\hline & \\
\hline-1.1217 & $(\mathrm{PP}(\mathrm{in})(\mathrm{NP}(\mathrm{NP}(\mathrm{effect}))))$ \\
\hline-1.1634 & $(\mathrm{VP}($ yield $)(\mathrm{PP}(\mathrm{PP}))(\mathrm{PP}($ in $)))$ \\
\hline-1.3574 & $(\mathrm{NP}(\mathrm{PP}($ in $)(\mathrm{NP}(\mathrm{NN}($ way $)))))$ \\
\hline-1.8030 & $(\mathrm{NP}(\mathrm{PP}($ in $)(\mathrm{NP}($ trading $)(\mathrm{JJ}))))$ \\
\hline \multicolumn{2}{|r|}{ shallow parsing } \\
\hline$w$ & active trees that contain the phrase "SBAR" \\
\hline 1.4500 & $(\operatorname{SBAR}(\mathrm{IN}($ for $))(\mathrm{NP}(\mathrm{VP}(\mathrm{TO}))))$ \\
\hline 0.6177 & $(\mathrm{VP}(\mathrm{SBAR}(\mathrm{NP}(\mathrm{VBD})))$ \\
\hline 0.6173 & $(\operatorname{SBAR}(\mathrm{NP}(\mathrm{VP}(“))))$ \\
\hline 0.5644 & $(\mathrm{VP}(\mathrm{SBAR}(\mathrm{NP}(\mathrm{VP}(\mathrm{JJ})))))$ \\
\hline-0.9034 & $(\operatorname{SBAR}(\mathrm{IN}($ for $))(\mathrm{NP}(\mathrm{O})))$ \\
\hline-0.9181 & $(\mathrm{SBAR}(\mathrm{NP}(\mathrm{O})))$ \\
\hline-1.0695 & $(\mathrm{ADVP}(\mathrm{NP}(\mathrm{SBAR}(\mathrm{NP}(\mathrm{VP})))))$ \\
\hline-1.1699 & $(\mathrm{SBAR}(\mathrm{NP}(\mathrm{NN})(\mathrm{NP})))$ \\
\hline
\end{tabular}

Table 4: Examples of active features (subtrees) All trees are represented in S-expression. In the shallow parsing task, $\mathbf{O}$ is a special phrase that means "out of chunk".

select relevant subtrees and achieves the best results for WSJ parsing. However, these techniques are not based on the regularization framework focused on this paper and do not always eliminate all the redundant subtrees. Even using the methods of (Bod, 2001), millions of subtrees are still exploited, which leads to inefficiency in real problems.

\section{Conclusions}

In this paper, we presented a new application of boosting for parse reranking, in which all subtrees are potentially used as distinct features. Although this set-up greatly increases the feature space, the $l_{1}$-norm regularization performed by boosting selects a compact and relevant feature set. Our model achieved a comparable or even better accuracy than kernel methods even with an extremely small number of features (subtrees).

\section{References}

Kenji Abe, Shinji Kawasoe, Tatsuya Asai, Hiroki Arimura, and Setsuo Arikawa. 2002. Optimized substructure discovery for semi-structured data. In Proc. of PKDD, pages 1-14.

Rens Bod. 1998. Beyond Grammar: An Experience Based Theory of Language. CSLI Publications/Cambridge University Press.
Rens Bod. 2001. What is the minimal set of fragments that achieves maximal parse accuracy? In Proc. of $A C L$, pages 66-73.

Eugene Charniak. 2000. A maximum-entropy-inspired parser. In Proc. of NAACL, pages 132-139.

Michael Collins and Nigel Duffy. 2002. New ranking algorithms for parsing and tagging: Kernels over discrete structures, and the voted perceptron. In Proc. of $A C L$.

Michael Collins. 1999. Head-Driven Statistical Models for Natural Language Parsing. Ph.D. thesis, University of Pennsylvania.

Michael Collins. 2000. Discriminative reranking for natural language parsing. In Proc. of ICML, pages 175-182.

Michael Collins. 2002. Ranking algorithms for named-entity extraction: Boosting and the voted perceptron. In Proc. of ACL, pages 489-496.

Yoav Freund, Raj D. Iyer, Robert E. Schapire, and Yoram Singer. 2003. An efficient boosting algorithm for combining preferences. Journal of Machine Learning Research, 4:933969.

Taku Kudo and Yuji Matsumoto. 2001. Chunking with support vector machines. In Proc. of NAACL, pages 192-199.

Taku Kudo and Yuji Matsumoto. 2004. A boosting algorithm for classification of semi-structured text. In Proc. of EMNLP, pages 301-308.

Simon Perkins, Kevin Lacker, and James Thiler. 2003. Grafting: Fast, incremental feature selection by gradient descent in function space. Journal of Machine Learning Research, 3:1333-1356.

Gunnar. Rätsch. 2001. Robust Boosting via Convex Optimization. Ph.D. thesis, Department of Computer Science, University of Potsdam.

Robert E. Schapire, Yoav Freund, Peter Bartlett, and Wee Sun Lee. 1997. Boosting the margin: a new explanation for the effectiveness of voting methods. In Proc. of ICML, pages $322-330$.

Fei Sha and Fernando Pereira. 2003. Shallow parsing with conditional random fields. In Proc. of HLT-NAACL, pages 213-220.

Erik F. Tjong Kim Sang and Sabine Buchholz. 2000. Introduction to the CoNLL-2000 Shared Task: Chunking. In Proc. of CoNLL-2000 and LLL-2000, pages 127-132.

Vladimir N. Vapnik. 1998. Statistical Learning Theory. WileyInterscience.

Guizhen Yang. 2004. The complexity of mining maximal frequent itemsets and maximal frequent patterns. In Proc. of SIGKDD.

Mohammed Zaki. 2002. Efficiently mining frequent trees in a forest. In Proc. of SIGKDD, pages 71-80.

Tong Zhang, Fred Damerau, and David Johnson. 2002. Text chunking based on a generalization of winnow. Journal of Machine Learning Research, 2:615-637. 